\title{
Search for neutrinos from Fast Radio Bursts with ANTARES
}

\author{
Damien Turpin \\ Université de Toulouse; UPS-OMP; IRAP; Toulouse, France \\ CNRS; IRAP; 14, avenue Edouard Belin, F-31400 Toulouse, France \\ E-mail: damien.turpin@irap.omp.eu

\section{Damien Dornic} \\ CPPM, Aix-Marseille Université, CNRS/IN2P3, 13009 Marseille, France \\ E-mail: dornic@eppm.in2p3.fr
}

\author{
Alexis Coleiro \\ APC, Univ Paris Diderot, CNRS/IN2P3, CEA/Irfu, Obs de Paris, Sorbonne Paris Cité, France \\ IFIC - Instituto de Física Corpuscular (CSIC - Universitat de València) c/ Catedrático José \\ Beltràn, 2 E-46980 Paterna, Valencia, Spain \\ E-mail: alexis.coleirodific.uv.es
}

\section{Matteo Sanguinetti*}

Universitá degli Studi di Genova, INFN Genova

E-mail: matteo.sanguineti@ge.infn.it

\section{on the behalf of the ANTARES collaboration and the SUPERB collaboration}

Fast Radio Bursts are one of the most mysterious transient sources. They are characterised by an intense radio-pulse lasting few milliseconds and mainly detected in the GHz energy band. Many unknowns remain concerning the nature of the transient progenitor, the nature of the radio emission and their distribution in the Universe. Recently, the first evidence on the association between the repeating burst FRB121102 and a star-forming dwarf galaxy located at the cosmological distance $\mathrm{z}=0.19$ was reported. These observations imply that at least some of the fast radio bursts indeed originate from the distant Universe and have to be associated with extremely violent events to explain their observed brightness. So far, the radiative processes powering the radio emission are unknown but efficient particle acceleration may occur in the vicinity of the progenitor. A multi-wavelength and a multi-messenger approach are therefore crucial to identify the nature of these acceleration mechanisms. In this context, a search for a high-energy neutrino signal from the most recent radio bursts has been performed with the ANTARES neutrino telescope. By design, ANTARES mainly observes the Southern sky ( $2 \pi$ steradian at any time) and is perfectly suited to search for a neutrino signal from sources of transients that have been mainly detected at the Parkes observatory in Australia. In this contribution, we will present the results of our searches with ANTARES and their implications for hadronic models of FRBs.

35th International Cosmic Ray Conference — ICRC2017

10-20 July, 2017

Bexco, Busan, Korea

\footnotetext{
* Speaker.
} 


\section{Introduction}

Energetic transient sources are promising candidates for a high-energy neutrino (TeV-PeV) detection as they would originate from explosive processes that release a huge amount of energy in a short timescale and heat the surrounding environment in violent shocks. In the shock fronts, the acceleration of hadrons could be efficient enough to explain the origin of the most energetic cosmic rays and the cosmological high-energy neutrinos discovered few years ago by the IceCube Collaboration [1]. In addition, the shorter the transient duration is the more significant will be the detection since typically the searches for a neutrino signal from a transient event below the minute timescale can be almost considered as background free and hence, the detection of a single neutrino event might be enough to claim a significant association with the transient source. The Gamma-ray Bursts (GRB) are one of the best example of such promising target for point like-source neutrino searches but without any success so far [2, 3, 4].

A decade ago, the Fast Radio Bursts (FRB) have been serendipitously discovered in the radio domain [5] and, up to now, constitute one of the most intriguing fast transient sources. They are characterised by a very bright radio-pulse (few Jy.s) lasting only few milliseconds and even less than a millisecond which make them the brightest radio source in the sky during their short lifetime. The measure of the uncommonly large delay between the high and the low radio frequencies, due to the dispersion of the radio signal by the column of the cold plasma crossed by the radio wave, points out toward an extragalactic and even a cosmological origin for these transients. This has been only confirmed for FRB121102 [6, 7, 8] and from the dispersion measures (DM) the cosmological distance distribution of FRBs is found in the range of redshift: $\mathrm{z}_{D M}=[0.19-1.4] \mathrm{ac}-$ cording to the public FRB catalogue ${ }^{1}$. To explain both the short timescale and the large energy budget of the FRBs they may be associated to extremely energetic events possibly to cataclysmic phenomena such as NS-NS merger [9, 10], short Gamma-ray Bursts (SGRB) [11, 12], the collapse of supramassive neutron star (SMNS) $[13,14]$ or maybe to giant flaring activities from highly magnetised neutron stars [15]. In the close environment of these extreme objects, hadronic acceleration processes may occur and would also lead to the production of TeV-PeV neutrinos mainly through photo-hadronic interactions between the ambient energetic photon field (typically x-ray/gammaray photons) produced by different radiative processes and the accelerated protons at a minimum energy $E_{p}>>\mathrm{GeV}$. Because of their remarkable transient properties similar to the Gamma-ray Bursts, if FRBs are indeed cosmic accelerators of hadrons, they offer a great opportunity for an unambiguous detection of cosmic neutrinos by the large neutrino telescopes.

The progenitors of the FRBs are still unidentified mainly because they have been only detected in the radio domain despite active searches at different wavelength $[16,17,18]$. Note that the observation of a $\gamma$-ray transient source associated to FRB131104 with the Swift satellite but under quite low significance $(3.2 \sigma)$ was reported by [19]. In such observational context, the multi-messenger observations with the high-energy neutrino telescopes can help to solve the "FRB mystery" and offer an unique way to address the following question : could the FRB progenitor be efficient cosmic accelerator of hadrons ? As a consequence, a search for a high-energy neutrino signal from the

\footnotetext{
${ }^{1}$ seehttp://www.astronomy.swin.edu.au/pulsar/frbcat/
} 
most recent FRBs has been performed with the ANTARES neutrino telescope [20]. The results are presented in this contribution.

\section{The FRB sample}

During the period 2013-2017, 12 FRBs have been detected by Australian radio facilities in different narrow bands such as the Parkes $\left(v_{o b s} \sim[1.2-1.5] \mathrm{GHz}\right)$, the ASKAP $\left(v_{o b s} \sim[0.7-1.8]\right.$ $\mathrm{GHz})$ and the UTMOST $\left(v_{o b s} \sim[0.83-0.85] \mathrm{GHz}\right)$ telescopes. Among them, 9 FRBs were visible by ANTARES at the trigger time and the detector was taking data in good conditions. Below we describe the characteristics of these 9 FRBs.

Table 1: Properties of the 9 FRBs visible by ANTARES in the period 2013-2017

\begin{tabular}{cccccc}
\hline FRB & $z_{D M}$ & date (UTC) & RA $\left(^{o}\right)$ & $\operatorname{dec}\left({ }^{o}\right)$ & radio trigger \\
\hline \hline & & & & & \\
131104 & 0.59 & $2013-11-0418: 03: 59$ & 101.04 & -51.28 & Parkes \\
140514 & 0.44 & $2014-05-1417: 14: 09$ & 338.52 & -12.31 & Parkes \\
150215 & 0.55 & $2015-02-1520: 41: 41$ & 274.36 & -4.90 & Parkes \\
150418 & 0.49 & $2015-04-1804: 29: 04$ & 109.15 & -19.01 & Parkes \\
150807 & 0.59 & $2015-08-0717: 53: 55$ & 340.10 & -55.27 & Parkes \\
160317 & 0.70 & $2016-03-1708: 30: 58$ & 118.45 & -29.61 & UTMOST \\
160410 & 0.18 & $2016-04-1008: 16: 54$ & 130.35 & 6.08 & UTMOST \\
160608 & 0.37 & $2016-06-08 ~ 03: 52: 24$ & 114.17 & -40.78 & UTMOST \\
170107 & 0.48 & $2017-01-0720: 05: 45$ & 170.79 & -5.02 & ASKAP \\
& & & & & \\
\hline
\end{tabular}

\section{Searching method}

The analysis focused on the data collected during the acquisition period $\left[\mathrm{T}_{0}-6 \mathrm{~h} ; \mathrm{T}_{0}+6 \mathrm{~h}\right]$ where $T_{0}$ is the trigger time of the considered FRB. The search for a significant neutrino flux is based on the detection of up-going muon-track events spatially and temporally coincident with the radio burst emission. For each FRB, a search cone of $2^{\circ}$, corresponding to the ANTARES point spread function at $3 \sigma$, is set around the FRB position (for which the error on the position is negligible wrt to the ANTARES PSF). To remove a significant contamination of mis-reconstructed muon background events in the neutrino sample, selection cuts are applied event by event on quality variables of the track reconstruction algorithm: the local zenith angle, $\theta$, the error estimate of the reconstructed direction $\beta$ and the quality parameter of the reconstruction algorithm, $\Lambda$. We require that each selected up-going event $(\cos \theta>0)$ has a direction error $\beta<1^{\circ}$. The selection criterion on $\Lambda$ has been optimised FRB per FRB to give a potential discovery of $3 \sigma$ for one neutrino event in a searching time window of $\Delta T=\left[\mathrm{T}_{0}-6 \mathrm{~h} ; \mathrm{T}_{0}+6 \mathrm{~h}\right]$. This relatively large time window allows us to cover different scenarios for the delay between the radio and the possible neutrino emission with a high potential of discovery.

Before applying the searching method to the sample of FRBs, the detector stability has been 
checked by looking at the total event rates detected in time slices of $2 \mathrm{hrs}$ within $\Delta T_{\text {back }}=\left[\mathrm{T}_{0}-6 \mathrm{~h}\right.$; $\left.\mathrm{T}_{0}+6 \mathrm{~h}\right]$. No significant variability was found in the event rates which guarantees the stability of the detector.

We finally found no up-going events temporally and spatially correlated with the 9 selected FRBs. Then, the number of atmospheric background events, $\mu_{b}$, has been directly estimated from the data using the $\Delta T_{\text {back }}$ time window. The expected number of background events in a ROI of $2^{\circ}$ is found to be $\mu_{B} \sim 5 \cdot 10^{-8}$ event $\cdot \mathrm{s}^{-1}$ which corresponds to a Poisson probability of observing zero event knowing the background noise, $\mu_{b}, P \geq 99 \%$. Hence, the null result is compatible with the neutrino background expectation.

\section{Constraints on the neutrino emission from FRBs}

As no significant neutrino signal has been detected from the selected FRBs by ANTARES, we can set upper limits on the neutrino fluence, $F_{v}$, that would yield to a detection at $90 \%$ C.L. First of all, the observable quantity that can be constrained with ANTARES data is the expected number of neutrinos, $\mathrm{N}_{v}$, that directly depends on the acceptance of the detector, $\operatorname{Acc}(\delta)$ and the neutrino flux. As the neutrino emission time is unknown, the instantaneous ANTARES acceptance was estimated at the FRB trigger times and is valid for all time in the search window. Then, $\mathrm{N}_{v}$ mainly depends on the declination coordinate $(\delta)$ of the source and its spectral model, $\frac{\mathrm{dN}_{\mathrm{N}}}{\mathrm{E}_{v}}=\phi \mathrm{E}_{v}^{-\mathrm{p}}$. In this analysis, two source models have been tested, a soft one with a spectral index $\mathrm{p}=2$ and a hard spectrum model with $\mathrm{p}=1$. According to the Poisson statistics, a neutrino signal of $\mathrm{N}_{v}=2.3$ events gives a 90\% C.L upper limit on the flux normalisation factor given by $\phi_{90 \%}=\mathrm{N}_{v} / \operatorname{Acc}(\delta)$. Then, the corresponding $90 \%$ C.L. neutrino fluence upper limit, $F_{V, 90 \%}$, can be expressed as:

$$
\mathrm{F}_{v, 90 \%}=\int_{\mathrm{E}_{\min }}^{\mathrm{E}_{\max }} \frac{\mathrm{dN}}{\mathrm{dE}_{v}} \cdot \mathrm{E}_{v} \cdot \mathrm{dE}_{v} \text { in erg } \cdot \mathrm{cm}^{-2}
$$

where $E_{\min }$ and $E_{\max }$ are the energies that define the 5-95\% range of the energy distribution of events passing the applied quality criteria $\left(\cos \theta>0\right.$ and $\left.\Lambda_{c u t}, 3 \sigma+\beta<1^{\circ}\right)$ for the corresponding spectrum.

To compute such upper limits we used Monte-Carlo simulations to reproduce the data taking conditions of the detector at each FRB trigger time. A sample of neutrino MC events is therefore modeled and the event times have been set at the FRB trigger time (instantaneous limits). The equatorial coordinates of each event (RA, Dec) are computed from the simulated local coordinates and the event times. Then, each event of energy, $\mathrm{E}_{v}$, passing the cuts $\left(\beta<1^{\circ}\right.$ and $\left.\Lambda>\Lambda_{\text {cut }, 3 \sigma}\right)$ contributes to the neutrino flux with a weight given by the instantaneous ANTARES acceptance.

The results on the neutrino fluence upper limits, $F_{v, 90 \%}$, for the two considered neutrino spectra are given in table 2 .

The fluence upper limits can be converted into a constraint on the isotropic total energy released in neutrinos in the rest frame according to the equation 4.2:

$$
E_{v, 90 \%}^{\text {iso }}=4 \pi D(z)^{2} \times F_{v, 90 \%} /(1+z)
$$


Table 2: Upper limit on the neutrino fluence, $F_{v, 90 \%}$ (given in unit of $\mathrm{erg} \cdot \mathrm{cm}^{-2}\left(\mathrm{GeV} \cdot \mathrm{cm}^{-2}\right)$ ), estimated for the 9 selected FRBs according to the instantaneous ANTARES sensitivity. The limits are given where $90 \%$ of the neutrino signal is expected between $\left[E_{\min }-E_{\max }\right]$ given in $\log _{10}[\mathrm{GeV}]$.

\begin{tabular}{|c|lc||lc||c|}
\hline & \multicolumn{3}{|c||}{$E^{-2}$} & \multicolumn{2}{c||}{$E^{-1}$} \\
FRB & $F_{V, 90 \%}$ & {$\left[\mathrm{E}_{\min }-\mathrm{E}_{\max }\right]$} & $F_{v, 90 \%}$ & {$\left[\mathrm{E}_{\min }-\mathrm{E}_{\max }\right]$} & $\Lambda$ cut \\
\hline 131104 & $1.4 \cdot 10^{-2}(8.8)$ & {$[3.4-6.8]$} & $20.7 \cdot 10^{-1}(1294)$ & {$[5.8-7.9]$} & -5.52 \\
140514 & $2.3 \cdot 10^{-2}(14.4)$ & {$[3.6-6.9]$} & $45.0 \cdot 10^{-1}(2805)$ & {$[5.8-7.9]$} & -5.50 \\
150215 & $2.8 \cdot 10^{-2}(17.7)$ & {$[3.1-6.5]$} & $41.4 \cdot 10^{-1}(2583)$ & {$[5.8-7.9]$} & -5.56 \\
150418 & $2.1 \cdot 10^{-2}(13.2)$ & {$[3.5-6.9]$} & $24.1 \cdot 10^{-1}(1502)$ & {$[5.8-8.0]$} & -5.52 \\
150807 & $1.9 \cdot 10^{-2}(12.2)$ & {$[3.6-6.9]$} & $5.4 \cdot 10^{-1}(339)$ & {$[5.8-8.0]$} & -5.60 \\
160317 & $2.1 \cdot 10^{-2}(12.8)$ & {$[3.5-6.9]$} & $42.6 \cdot 10^{-1}(2659)$ & {$[5.8-7.9]$} & -5.58 \\
160410 & $1.9 \cdot 10^{-2}(11.8)$ & {$[3.6-6.9]$} & $7.1 \cdot 10^{-1}(444)$ & {$[5.8-7.9]$} & -5.56 \\
160608 & $2.6 \cdot 10^{-2}(16.3)$ & {$[3.6-7.0]$} & $24.3 \cdot 10^{-1}(1516)$ & {$[5.8-7.9]$} & -5.68 \\
170107 & $1.4 \cdot 10^{-2}(8.8)$ & {$[3.5-6.9]$} & $4.3 \cdot 10^{-1}(267)$ & {$[5.7-7.9]$} & -5.58 \\
\hline
\end{tabular}

where $\mathrm{z}$ is the redshift of the source. The distance of the neutrino source, $\mathrm{D}(\mathrm{z})$, is computed as follows :

$$
D(z)=\frac{c}{H_{0}} \int_{0}^{z} \frac{\left(1+z^{\prime}\right) d z^{\prime}}{\sqrt{\Omega_{m}\left(1+z^{\prime}\right)^{3}+\Omega_{\Lambda}}}
$$

where $\Omega_{m}=0.308$ and $\Omega_{\Lambda}=0.692, H_{0}=67.80 \mathrm{~km} \cdot \mathrm{s}^{-1} \cdot \mathrm{Mpc}^{-1}$ following [21] and c is the light speed. As the distance of each FRB is poorly constrained, we test different kind of scenarios : a galactic origin $(\mathrm{D} \in[1-50] \mathrm{kpc})$, an extragalactic origin $(\mathrm{D} \in[0.05-100] \mathrm{Mpc})$ and a cosmological origin $\left(\mathrm{z} \in\left[0.02-\mathrm{z}_{D M}\right]\right)$. The $90 \%$ upper limits on $E_{v, 90 \%}^{\text {iso }}$ for the two neutrino spectra, $E^{-2}$ and $E^{-1}$, are shown in the figures 1 and 2 .

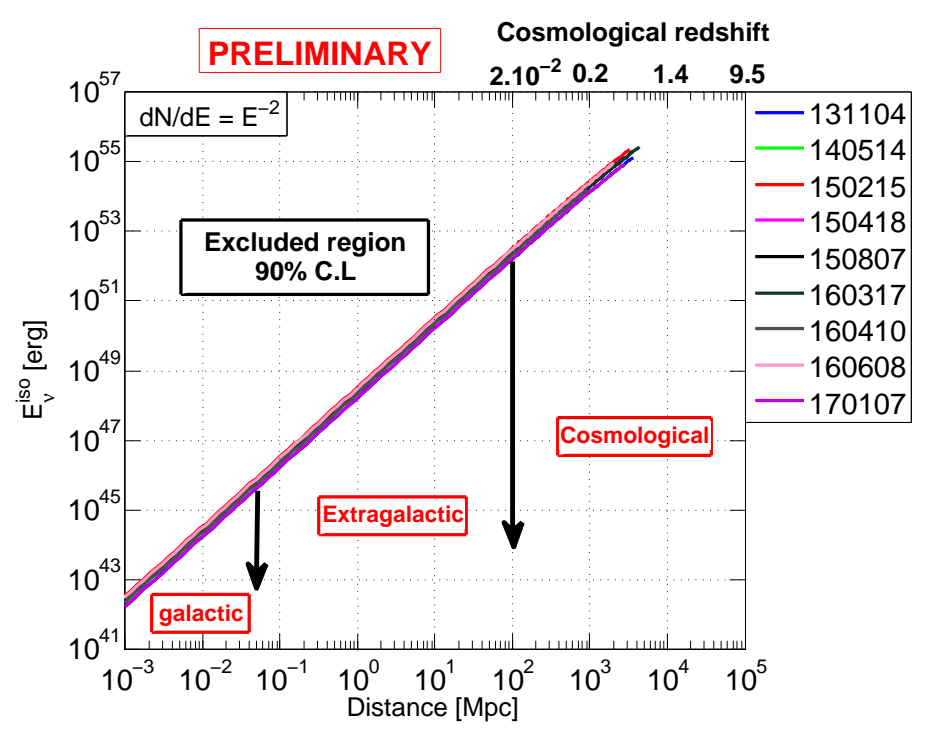

Figure 1: Upper limits on the neutrino energy released by the 9 selected FRBs as function of their plausible distances assuming a $E^{-2}$ spectrum. 


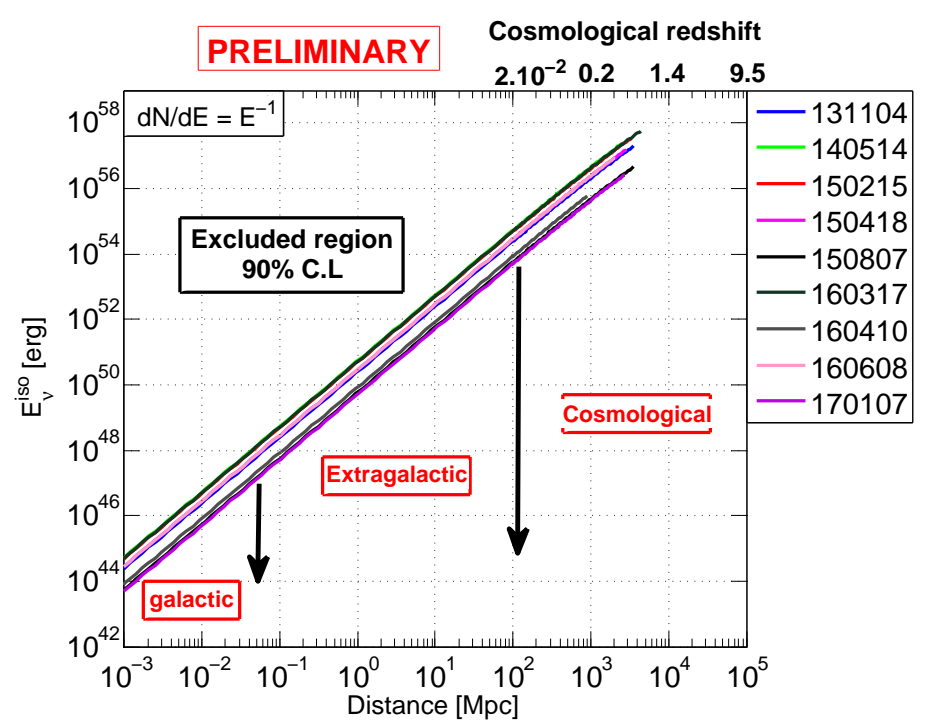

Figure 2: Same as the figure 1 assuming a $E^{-1}$ spectrum.

If these FRBs are neutrino emitters, ANTARES puts interesting constraints on their plausible origin. Indeed, at distances d $\lesssim 50 \mathrm{Mpc}$, the ANTARES constraints, for a $E^{-2}$ source model, are at the level of $\mathrm{E}_{v, 90 \%}^{\mathrm{iso}} \lesssim 10^{52} \mathrm{erg}$. In the case of a $E^{-1}$ spectrum, the energy is mainly deposited in the $\mathrm{PeV}$ domain where the ANTARES sensitivity is largely degraded by the Earth absorption. Thus, the limits on $\mathrm{E}_{v, 90 \%}^{\mathrm{iso}}$ are lowered by an order of magnitude. In any spectral case, the cosmological scenario is poorly constrained by ANTARES.

\section{Conclusion}

In this contribution, the first search into the ANTARES data for a high-energy neutrino signal from a population of Fast Radio Bursts detected these last four years has been reported. No neutrino events were spatially found in correlation with the nine selected FRBs in a time window extended up to six hours before and after the radio bursts. These non detections allow us to derive 90\% C.L. upper limits on the neutrino fluence at the FRB trigger times. As the neutrino production during a FRB event is largely unknown, the limits were derived assuming standard hard and soft power law neutrino spectra with spectral indexes $\mathrm{p}=1$ and 2, respectively. The limits are of the order of $\mathrm{F}_{v, 90 \%} \lesssim 10^{-1}-10^{-2} \mathrm{erg} \cdot \mathrm{cm}^{-2}$ for a $E^{-1}$ and $E^{-2}$ source model, respectively. Since the FRB distances are poorly constrained, the corresponding isotropic energies released in neutrinos were then computed under different distance hypothesis: a galactic, an extragalactic or a cosmological origin. The constraints on $\mathrm{E}_{v, 90 \%}^{\mathrm{iso}}$ for different distances are given in the figures 1 and 2. First, it is clear that the ANTARES sensitivity is too low to significantly constrain any cosmological scenario for the FRB progenitors $\left(\mathrm{E}_{v, 90 \%}^{\mathrm{iso}} \lesssim 10^{55}-10^{54}\right.$ erg for $\mathrm{z} \sim 0.1$ for a $E^{-1}$ and $E^{-2}$ source model, respectively). However, the galactic and very close extragalactic scenarios $(\mathrm{D}<1$ $10 \mathrm{Mpc}$ ) start to be interestingly restricted below $\mathrm{E}_{v, 90 \%}^{\text {iso }} \sim 10^{52} \mathrm{erg}$. To go further into the FRB neutrino model constraints, larger scale neutrino detectors $\left(\mathrm{km}^{3}\right)$ are needed to catch the neutrino signal from the potential FRB progenitors such as giant flares from magnetars or short GRBs. This 
might be achievable in the next few years with the new generation of european neutrino telescopes KM3NeT/ARCA that will be 50 times more sensitive than ANTARES [22].

\section{References}

[1] The IceCube Collaboration, 2013, Science, 342

[2] Abbasi, R., Abdou, Y., Abu-Zayyad, T., et al. 2012, Nature, 484, 351

[3] Adrián-Martínez, S., Albert, A., Samarai, I. A., et al. 2013, A\&A, 559, A9

[4] Ackermann, M. and Adams, J. and Aguilar, J. A. and Ahlers, M., et al. 2016, ArXiv e-prints : arXiv1601.06484

[5] Lorimer et al., 2007, Science, 318, 777

[6] Tendulkar, S. P., Bassa, C. G., Cordes, J. M., et al. 2017, ApJL, 834, L7

[7] Chatterjee, S., Law, C. J., Wharton, R. S., et al. 2017, Nature, 541, 58

[8] Marcote, B., Paragi, Z., Hessels, J. W. T., et al. 2017, ApJL, 834, L8

[9] Totani, T. 2013, PASJ, 65

[10] Wang, J.-S., Yang, Y.-P., Wu, X.-F., Dai, Z.-G., \& Wang, F.-Y. 2016, ArXiv e-prints : $\operatorname{arXiv1603.02014}$

[11] Zhang, B. 2014, ApJL, 780, L21

[12] Palaniswamy, D., Wayth, R. B., Trott, C. M., et al. 2014, ApJ, 790, 63

[13] Ravi, V. \& Lasky, P. D. 2014, MNRAS, 441, 2433

[14] Li, X., Zhou, B., He, H.-N., Fan, Y.-Z., \& Wei, D.-M. 2014, ApJ, 797, 33

[15] Popov, S. B. \& Postnov, K. A. 2013, ArXiv e-prints : arXiv1307.4924

[16] Petroff, E., Keane, E. F., Barr, E. D., et al., 2015, MNRAS, 451, 3933

[17] H. E. S. S. Collaboration and Abdalla, H., Abramowski, A., Aharonian, F., et al., 2017, A\&A, 597, A115

[18] Petroff, E. and Burke-Spolaor, S. and Keane, et al., 2017, MNRAS, 469, 4465-4482

[19] DeLaunay, J. J., Fox, D. B. and Murase, K. and Mészáros, P., et al., 2016, ApJL, 832, L1

[20] Ageron, M., Aguilar, J. A., Al Samarai, I., et al. 2011, Nuclear Instruments and Methods in Physics Research A, 656, 11

[21] Planck Collaboration, Ade, P. A. R., Aghanim, N., et al., 2016, A\&A, 594, A13

[22] Adrián-Martínez, S., Ageron, M., Aharonian, F., et al. 2016, ArXiv e-prints : arxiv1601.07459 